\title{
Maud Graham et l'affaire de la mauvaise mère : le polar féministe chez Chrystine Brouillet (1987-2007)
}

\author{
Kathleen Kellett-Betsos \\ Université Ryerson
}

L'auteure québécoise Chrystine Brouillet affirme avoir créé le personnage de Maud Graham pour contrer une certaine vision de la féminité dans le roman policier traditionnel: «J'en avais assez de voir des stéréotypes de femmes dans certains de ces romans, les blondes pulpeuses, aguichantes n'ont rien à voir avec notre réalité. Je suis féministe, je ne vois pas comment on peut ne pas l'être.» (citée par Godin, 1992-1993, p.34) Or, parmi les stéréotypes féminins de ce genre énumérés par Anne Lemonde dans La Femme et le roman policier se trouve celui de la «femme-refuge », représentée par la mère, l'épouse ou par 
tout autre personnage maternel qui évoquent un monde sécuritaire et tranquille, faisant contraste avec le milieu criminel et violent où circule le détective ${ }^{1}$ (Lemonde, 1984, p. 39). Chez Brouillet, l'image de la femme-refuge se brise; la mère peut être héroïque, mais aussi incompréhensive, égoïste et même criminelle. Brouillet met en question le rôle traditionnel de la mère surtout en posant son personnage principal, la détective Maud Graham, comme figure maternelle alternative, venue réparer les dégâts occasionnés par une structure familiale contrainte par une dynamique de pouvoir déséquilibrée. En fait, Graham en vient à remplir le rôle d'über-mère dans une version renouvelée de la famille. Dans The Woman Detective, Kathleen Klein remarque le paradoxe du polar féministe: alors que le féminisme devrait s'attaquer au statu quo, la structure du roman policier exige la résolution d'un mystère, généralement une affaire de meurtre. Si le détective est policier, comme c'est le cas avec Maud Graham, il a le devoir de remettre le coupable entre les mains de la justice. Le détective rétablit donc l'ordre social perturbé par l'acte criminel (Klein, 1995 [1988], p. 200201). Auteure de Murder by the Book? Feminism in the Crime Novel, Sally Munt souligne les limites du roman policier, caractérisé par le féminisme libéral qui se focalise normalement sur la réforme des structures régissant la famille, ce bastion de la société occidentale (1994, p. 43). Elle cite l'exemple de la détective V.I. Warshawski chez l'auteure américaine Sara Paretsky :

The family is a crucial site of interest for liberal philosophy, straddling as it does the individual and the state. [...] Within the structure of the novels, the threat to the family is always removed by Warshawski, the enigma resolved, and order

${ }^{1}$ Lemonde emprunte ce terme à Lacombe (1975, p. 149). 
restored. This effectively re-forms it (in both senses) and perpetuates a mildly revisionist status quo, so that Warshawski is complicit with its continuing hegemony ${ }^{2}$. (p. 34)

Nous verrons que Maud Graham, autre féministe libérale, partage cette attitude protectrice envers la famille tout en s'intéressant particulièrement au rôle de la mère, traditionnellement centre émotif de la famille nucléaire.

En tant qu'agente de police, Maud Graham est réformiste plutôt que révolutionnaire : elle vise à transformer la société en accédant aux structures du pouvoir afin de les réformer. Dans les dix romans de la série, depuis Le Poison dans l'eau, paru en 1987, jusqu'à Promesses d'éternité, paru en 2009, son souci pour les personnages démunis et marginalisés reste constant. Le narrateur de Préférez-vous les icebergs? souligne les intentions cachées de la policière : « Mais Graham avait triché dès le début; elle avait une âme d'assistante sociale qui se serait déguisée en flic pour avoir davantage de pouvoir.» (1988, p. 70) Si elle maugrée lorsque son patron lui assigne surtout des cas d'inceste et de viol, perçus comme relevant de la vie privée et donc du domaine féminin, Graham profite néanmoins de l'occasion pour materner les victimes. Par exemple, elle exige de son supérieur son propre bureau en lui expliquant que les femmes battues ont besoin de parler derrière des portes closes : «Graham sait qu'on a accédé à sa requête pour confiner ce genre de problèmes dans un seul bureau. Pour une seule personne. N'empêche, elle peut maintenant fermer la porte sur

\footnotetext{
2 « La famille est un lieu d'intérêt crucial pour la philosophie libérale, car elle enjambe l'individu et l'État. [...] Au sein de la structure des romans, la menace faite à la famille est toujours éliminée par Warshawski, l'énigme résolue et l'ordre restauré. Ceci la ré-forme (dans les deux sens du terme) effectivement et perpétue un statu quo légèrement réformiste ainsi que Warshawski est complice de sa continuelle hégémonie. » (trad. Amy J. Ransom)
} 
les blessures de ses victimes. » (1987, p. 125) Dès le premier roman, elle établit un centre d'écoute pour les victimes de viol et d'inceste. Informée d'un manque de fonds pour établir ce centre, elle contourne les objections des autorités en faisant annoncer dans les journaux que l'argent avait déjà été accordé pour ce projet, ce que son patron ne veut pas nier face à la réception favorable du public envers cette initiative. Dans C'est pour mieux t'aimer, mon enfant (1996), on apprend que Graham a mis sur pied un centre pour femmes battues pour et les enfants de celles-ci et qu'elle organise une soirée-bénéfice pour soutenir ces projets.

L'attitude protectrice de Graham ne s'applique pas seulement aux femmes et aux enfants. Dans Le Collectionneur, lorsqu'un journaliste lui demande : «Allez-vous [...] combattre [le tueur] avec autant d'énergie s'il s'occupe maintenant des hommes? », elle s'offusque : «Elle faillit répondre qu'elle était féministe, oui, mais elle n'avait jamais eu pour autant envie de castrer les hommes et elle tenterait de les protéger de la même manière qu'elle protégeait les femmes. » (1995, p. 155) Graham est ici autant représentante de la loi que mère justicière déterminée à protéger ses enfants, les citoyens du Québec. Au cours de ses enquêtes, la détective féministe bute contre les aspects malsains de la dynamique familiale où la mère est sujette à des attentes irréalistes. Examinons les avatars de la maternité en fonction de certaines catégories relevées d'une lecture des dix romans mettant en vedette la détective Maud Graham : la mère porteuse ou la femme-réceptacle, la mère reine de beauté, la mère-pieuvre, la mère célibataire effacée et, finalement, l'übermère et sa famille non-traditionnelle. 


\section{La mère porteuse ou la femme-réceptacle}

En tant que féministe, Maud Graham met en question le rôle traditionnel de la femme comme mère et épouse. La mère de Graham même lui déconseille une carrière dans la police puisqu'elle la voit comme un obstacle au mariage (1996, p. 212). Dans Le Poison dans l'eau, Catherine boit pour oublier son effacement à l'intérieur de son propre foyer; son mari Philippe, qui l'empoisonne lentement comme il a empoisonné sa première femme, la considère comme une simple matrice, une mèreréceptacle qui lui a permis d'engendrer sa fille Emma, qu'il aime d'un amour incestueux. En visant sa femme, Philippe finit par tuer sa belle-fille, Julie-Anne, meurtre dont son fils Mathieu est accusé. Le père s'en réjouit : «Bien sûr. C'était une coïncidence incroyable, inespérée, parfaite... On trouvait du poison dans le corps, c'est toi qui étais condamné. J'aurais achevé Catherine. Emma est pour moi. Je l'ai faite pour moi! » (p. 207) Catherine croit que sa fille Emma, qu'elle n'aime guère, est responsable du meurtre, mais elle ne la dénonce pas avant que son beau-fils Mathieu ne soit accusé. Incapable de quitter son mari parce qu'elle serait sans le sou si elle divorçait, Catherine semble également avoir renoncé à son rôle maternel, se contentant de s'adonner à l'alcoolisme et aux liaisons adultères.

À première vue, Suzanne Geremelek, dans Les Fiancées de l'enfer (1999), représente aussi la mère-porteuse. Bouleversée par la mort de son fils Éric, âgé de quinze mois, elle sombre dans un monde de fantasmes au moyen de médicaments et de vols à l'étalage dans des boutiques pour bébés. Son mari la traite comme une enfant qu'il doit protéger et sa fille, aliénée, pratique l'auto-mutilation, se drogue et s'efforce d'entrer en 
contact avec son frère mort. Poursuivant son optique féministe, Brouillet situe la source des troubles psychologiques de Suzanne dans une adolescence vécue avec un père veuf qui abusait d'elle sexuellement et qui justifiait l'inceste en citant l'exemple de l'Égypte antique :

Le Dr Labonté avait expliqué à Suzanne que les rois égyptiens se mariaient entre eux : Cléopâtre avait épousé ses deux frères. [...] Ils [...] imitaient [les rois égyptiens], s'élevaient au-dessus du commun des mortels. Dans ses rêves, Joël Labonté flottait au-dessus d'elle, tel un dieu, omniprésent, occupant tout l'espace sans lui laisser le moindre interstice de liberté. (p. 78)

Obsédé par son désir pour l'adolescente pubère, le père a tendance à réifier le corps féminin. L'aliénation de Suzanne se manifeste dans la sensation de fragmentation de son corps :

Suzanne avait si fortement l'impression d'être fragmentée qu'il lui arrivait parfois de ressentir physiquement cet éclatement: un doigt ou le bout de son pied, sa fesse gauche, son œil droit manquaient de sensibilité quand elle les touchait. Comme si ces parties de son corps appartenaient à une autre femme. (p. 45)

Sans que son mari le sache, leur fille Marie-Ève est également la sœur de Suzanne. Négligée par sa mère, Marie-Ève est d'abord attirée par la bonté simulée de Joël Labonté. En surprenant son père en train de s'attaquer à Marie-Ève, Suzanne l'abat, mettant fin à la carrière de ce Barbe-Bleue, image qu'elle avait toujours évoquée; elle soupçonnait en effet son père d'avoir tué sa mère à elle. De mauvaise mère, Suzanne se transforme, après le parricide, en mère férocement protectrice: «Une certaine sérénité flottait dans son regard : la fierté d'une lionne qui a su protéger son petit et qui ne se souvient même pas du goût du sang de son ennemi dès que la vie revient à la normale. » (p. 218) 
Une autre piste narrative à l'intérieur des Fiancées de l'enfer illustre la façon dont le refus de l'autorité patriarcale par la mère-épouse peut déclencher une série d'actions destinées à remettre les femmes à leur place. Steeve [sic] Tremblay voudrait se venger du meurtre-suicide de son frère Paul, qui a tué sa propre femme et ses trois fils lorsque celle-ci a décidé de quitter son mari en exigeant la garde des enfants. Tremblay en veut surtout à Graham pour avoir encouragé Ginette à fuir son mari violent en se réfugiant dans le centre pour femmes et enfants en détresse que la détective a fait établir. Selon lui, si son frère a parfois "corrigé » sa femme en la battant, c'est qu'elle l'avait bien mérité (p. 56). Sa haine contre les femmes se traduit par une violence presque surréelle. Il viole ses victimes avec un godemiché avant de graver sur leur sein gauche l'initiale du nom de chacun des hommes de sa famille qui a péri dans le meurtre-suicide. Toutefois, il ne peut pas réussir son opération sur l'une des femmes, car celle-ci vient de subir une mammectomie. Son affolement révèle sa crainte du pouvoir castrateur de la femme : «Il détestait les surprises! Il n'aurait pu graver l'initiale de Paul dans la chair sans être obligé d'y toucher. Et si sa main avait glissé sur la plaie, dans la plaie, si les lèvres s'étaient décollées, s'étaient ouvertes pour avaler ses doigts, puis son bras? » (p. 148) Impatient de se venger contre la mère en défigurant le sein, métonymie du pouvoir maternel d'offrir, mais aussi de refuser, la nourriture vitale, Tremblay est bouleversé par ce décalage entre la femme fantasmée et la femme réelle. La révulsion inspirée par l'abject se rattache, selon Julia Kristeva, à l'ambivalence envers le corps maternel associé à la fusion préœdipienne qui offre la sécurité corporelle tout en menaçant d'engloutir l'enfant, d'effacer les frontières entre soi et l'autre (1980, voir notamment p. 65 et 94). Le corps 
maternel a un pouvoir qu'il faut mater si l'on veut préserver l'intégrité individuelle et maintenir le pouvoir masculin à l'intérieur de la famille patriarcale. À cet égard, les « corrections » administrées à la femme de Paul Tremblay ainsi que les viols commis par Steeve Tremblay relèvent du besoin pressant de contrôler la femme, dont le corps représente une menace potentielle à l'intégrité psychique.

\section{La mère reine de beauté}

L'obsession de l'image corporelle, aiguë chez les femmes dans notre société, représente chez Brouillet un obstacle à l'épanouissement personnel ainsi qu'à l'expression entière de l'amour maternel. La mère qui valorise trop la beauté est moins apte à offrir à son enfant le réconfort de l'amour inconditionnel. L'adolescente Betty Désilets, personnage récurrent depuis Les Indésirables (2003), est négligée par ses parents, surtout sa mère, belle et mince, qui accepte mal sa fille grassouillette couverte de boutons; son manque d'amour-propre amène Betty à se laisser séduire par un bel homme qui voudrait qu'elle le débarrasse de sa femme riche.

Les troubles de l'image corporelle inspirent des fantasmes freudiens d'une férocité sanglante dans $L e$ Collectionneur, où les pulsions criminelles d'un tueur en série relèvent directement d'un attachement œdipien à la mère, ancienne reine de beauté. Le tueur, Michaël Rochon, collectionne des parties du corps de ses victimes afin de créer un terrifiant mobile composé de trois figures : la mère, une figure composite couronnée de la tête de sa propre mère décapitée lors d'un accident de voiture; le fils, qui devrait être 
représenté par le corps empaillé d'un garçon de douze ans (l'âge qu'il avait lors du divorce de ses parents); et le père, réduit à sa plus simple expression : un pénis empaillé. Ce désir de recomposer la mère à partir des parties du corps de plusieurs femmes se rattache à la réification de la femme, implicite dans le discours de la mère de Rochon, qui parle constamment à son fils de son ancienne gloire de gagnante de concours de beauté :

Elle le faisait asseoir près d'elle et lui expliquait comment elle avait gravi les marches du podium, lentement, félinement, lascivement. Les juges n'avaient pas pu détacher leurs regards de ses jambes, de ses seins, de ses fesses si fermes! Pas comme celles de son fils...? (1995, p. 38-39)

Elle aurait voulu un fils aussi beau qu'Elvis Presley : "Cette belle gueule d'amour, et ce déhanchement! Et il aimait sa mère, Elvis; il l'avait gâtée dès qu'il avait commencé à gagner de l'argent. » (p. 39) Pour plaire à sa mère, Michaël participe à un concours de M. Univers, mais sans succès. Cet échec est compensé par le pouvoir qu'il ressent en tant que tueur en série : "Toutefois, il terrifiait une ville bien plus efficacement que ce M. Muscles. Et sans vraiment se servir de ses muscles. » (p.107) En hommage à sa mère, il voudrait faire tourner son mobile au rythme de la chanson d'Elvis, « Love me tender ».

Après avoir sauvé le jeune garçon que Rochon avait destiné au rôle du fils empaillé, Maud Graham assume une attitude maternelle en le berçant et en lui chantant une chanson douce. Frédéric sera remis à la garde du père autoritaire et de la mère effacée dont il s'était enfui. Graham explique les dangers encourus par le garçon en grande partie par la négligence maternelle; elle est donc contente d'apprendre que la mère de Frédéric a décidé de laisser tomber l'alcool et les pilules pour 
mieux s'occuper de son fils. Graham ne peut pas s'empêcher de condamner la mère du tueur non plus : «Francine Rochon n'a jamais témoigné d'affection à son fils et il a trouvé d'autres moyens d'attirer son attention. » (1995, p. 213) Lorsque son ami Grégoire proteste contre cette observation trop simpliste, Graham renchérit : "Je sais que ce ne sont pas tous les enfants mal aimés qui deviennent des tueurs en série. Mais l'inverse est vrai. Il faut le dire! Ça devrait faire réfléchir les parents qui ne s'occupent pas de leurs enfants. » (p. 213) Il est tout de même curieux que cette condamnation, dans ces romans, tombe aussi rarement sur les pères absents ou effacés.

La notion que la valeur de l'être humain, surtout la femme, réside dans un corps idéal, perçu comme un assemblage de beaux seins et de belles jambes, mène dans ce roman à la perspective déshumanisante du tueur. En même temps, en fragmentant le corps de la mère, le tueur en diminue la puissance. Le corps maternel, associé à l'abject par l'enfant, qui cherche l'individuation, est maîtrisé et modelé par Michaël Rochon. Le père, représenté par le phallus, est réduit à la plus simple expression de la masculinité. La théorie féministe, qui puise dans le discours psychanalytique, insiste sur l'image terrifiante de la mère toute-puissante, dont l'enfant dépend presque exclusivement, surtout en l'absence du père; la peur de la dépendance inspirerait un sentiment d'horreur, surtout chez le fils, qui aurait donc tendance à rabaisser la mère afin d'en limiter la puissance ${ }^{3}$. Réduite à un élément dans un beau collage de parties du corps, la mère de Michaël Rochon est transformée en poupée; le pouvoir du corps maternel est défait.

3 Voir Chodorow (1978), notamment p. 183, à propos du concept « the dread of the mother » chez la psychanalyste Karen Horney. 


\section{La mère-pieuvre}

Dans Soins intensifs (2000), Brouillet crée un personnage qui est l'incarnation même de la mère toute-puissante, qui se valorise en exigeant la dépendance totale de l'enfant. Graham a beaucoup de difficulté, au début, à déceler chez Denise Poissant les symptômes du syndrome de Münchhausen par procuration; celle-ci cherche à se faire valoir en participant aux soins médicaux subis par son fils Kevin, qu'elle a rendu malade ellemême de diverses façons. Denise Poissant se réjouit de pouvoir mystifier les médecins dans les hôpitaux où elle fait traiter son jeune fils. Ce qu'elle voudrait surtout, c'est qu'on pratique sur Kevin «une belle intervention» (p.45). La sollicitude apparente pour l'enfant qu'elle refuse de laisser seul à l'hôpital donne l'impression d'une mère dévouée, mais le narrateur révèle sa mauvaise foi, surtout dans des passages en discours indirect libre :

Elle s'était même abonnée à L'actualité médicale et la lisait toujours au complet, rêvant du jour où on $\mathrm{y}$ accepterait ses propres articles. Elle s'imaginait présenter un témoignage passionnant sur le cas de Kevin. N'était-elle pas la personne idéale pour exprimer avec des mots justes les nombreux traitements qu'avait subis l'enfant? (p. 21-22)

Graham souligne la nécessité de prouver la culpabilité de la mauvaise mère : «Vous direz ce que vous savez, mais l'image de la madone, de la mère aimante est gravée en nous. On vous croira avec des preuves. » (p. 176) Le dévouement apparent de Denise Poissant masque son rôle archétype de mère dévoratrice telle que l'entrevoit André Rouaix, le partenaire de Graham : "Il avait fait un cauchemar où elle avait un corps d'araignée et s'avançait vers lui pour l'emprisonner d'un fil 
gluant. Elle s'apprêtait à découper Kevin et il ne pouvait rien faire pour l'empêcher parce qu'il était collé dans sa toile. » (p. 216) Ayant dû renoncer à son ambition de devenir infirmière à la suite de son expulsion pour avoir signé de fausses ordonnances, ayant divorcé d'un homme qui n'était qu'un simple soldat alors qu'elle aurait voulu un héros décoré de médailles, Denise Poissant ne réussit à se faire valoir qu'en adoptant le rôle de mère vigilante auprès d'un enfant malade qu'elle seule peut guérir en cessant ses traitements pathogènes. Pour les lecteurs, sa mort doit paraître comme un exemple de justice romanesque. La personnalité obsessionnelle de Denise Poissant n'est qu'une image agrandie de la femme qui s'attire l'approbation sociale en remplissant son rôle de mère zélée.

\section{La mère célibataire effacée et la tentation du patriarcat}

On pourrait supposer que la rupture de la famille patriarcale apporterait un sentiment de libération pour tous ceux qui la composent. Cependant, il est évident, dans les romans de Brouillet, que les parents divorcés, et surtout les mères solitaires, souffrent de ne pas recevoir l'appui social dont ils ont besoin pour élever leurs enfants. Par exemple, dans Silence de mort (2008), la mère de Frank Potvin est une femme brisée par la vie qui élève son fils dans une maison aseptisée, dépourvue de chaleur, mais qui ne sait pas empêcher son implication dans le trafic de drogue et, finalement, sa mort. Dans Les Indésirables, il arrive à Graham de demander pourquoi les hommes divorcés acceptent aussi souvent de ne passer qu'un ou deux week-ends par mois avec leurs enfants, ce à quoi son partenaire Rouaix ne trouve pas de réponse satisfaisante (2003, p. 278). Graham est 
conséquente dans sa vision d'un État interventionniste qui aiderait les parents à subvenir aux besoins physiques et psychologiques des enfants.

Comme si l'éclatement de la famille nucléaire créait un vide social qu'il fallait combler, dans Promesses d'éternité, les fraudeurs Denis Tremblay et Martin Plante et le pyromane Carol Blondin-Warren profitent des familles en désarroi en créant la secte Espérance renouvelée. Dans un centre établi dans un motel abandonné, on accueille des démunis, à qui sont offerts des programmes de désintoxication. Les membres de la secte doivent se soumettre à l'autorité du Maître et de ses Gardiens. L'autorité paternelle traditionnelle est donc remplacée par celle du Maître, qui interprète pour les adeptes les vœux de Dieu. L'une des adeptes, Ariane Paradis, est une économiste bien rémunérée qui accepte de s'intégrer à la secte en y emmenant sa fille Marie-Lune. Selon Hélène Paradis, qui se reconnaît comme "une handicapée de l'amour maternel », sa fille Ariane est forte en affaires mais, ayant souffert d'un manque d'amour dans son enfance, elle est susceptible de succomber facilement aux charmes de beaux-parleurs tels que Martin Plante (2009, p. 75). Comme les autres parents, Ariane est séparée de sa fille et ne saurait donc la protéger du Gardien qui la séduit. Le Maître s'accorde le droit d'organiser des mariages pour les jeunes filles, souvent avec des adeptes bien plus âgés. Blondin-Warren en a d'ailleurs l'habitude : dans son ancienne secte, surnommée Le Ranch des vierges sacrifiées, les jeunes filles étaient agressées sexuellement par ses acolytes. La menace de l'Apocalypse plane sur tous les habitants du centre, puisque Blondin-Warren, atteint d'un cancer incurable, projette de les tuer et de se donner la mort dans un incendie 
spectaculaire qui aurait lieu le premier décembre, date d'anniversaire qu'il partage avec sa mère, Édith Blondin.

Femme effacée, victime de violence conjugale, Édith Blondin habite tranquillement dans un couvent près de Québec. Son fils Carol lui en veut pour son sentiment d'abandon lorsqu'elle a dû l'envoyer, enfant, chez son père aux États-Unis à la suite des incendies criminels dont il était responsable au Québec. Le frère d'Édith se rappelle l'incapacité de sa sœur de refuser quoi que ce soit à son fils, ce qui ne suffit sûrement pas à justifier la psychose de Carol, mais ce qui constitue un facteur de sa délinquance. De retour au Québec après la dissolution de la secte américaine, Blondin-Warren invite sa mère à se joindre à lui au centre. Il est furieux de son refus : "Il avait acquiescé, alors qu'il avait envie de lui crier qu'elle ne l'avait jamais aimé, qu'elle préférait être séparée de lui. » (2009, p. 374) Empêché par la police de mettre le feu au centre, il compte se suicider avec sa mère dans un incendie qui devait détruire le couvent et tous ses habitants. Cependant, ce projet criminel est déjoué par les actions de Maud Graham, justicière et über-mère, qui fait semblant d'être la mère de Blondin-Warren afin de désarmer le Maître incendiaire de l'Espérance renouvelée.

\section{Maud Graham : über-mère}

Ayant choisi de ne pas avoir d'enfants elle-même, Maud Graham joue le rôle de mère auprès des jeunes gens qu'elle rencontre dans sa vie professionnelle. Comme Sherlock Holmes avec son Dr Watson, Graham bénéficie d'un "personnage faire-valoir » (Lemonde, 1984, p. 48) : le jeune prostitué Grégoire, avec qui 
elle s'est liée d'amitié. Renvoyé de chez sa mère qui ne voulait pas croire que son concubin agresse son fils sexuellement, Grégoire offre à Graham sa capacité, aiguisée par le contact avec ses clients et des vendeurs de drogue, d'évaluer le caractère des suspects. Le rôle maternel de Graham se précise à partir de la première parution de Grégoire, dans le deuxième roman de la série. Dans C'est pour mieux t'aimer, mon enfant, lorsque son partenaire remarque que Grégoire est un ange, Graham le remercie du compliment, mais s'interroge aussitôt: «Merci de quoi? Grégoire n'était pas son fils, elle ne l'avait pas élevé. Elle avait pourtant l'impression d'être responsable de lui. » (p. 164) En général prompte à comprendre la faiblesse humaine, $\mathrm{y}$ compris celle des femmes «[é]puisées, déprimées [...] [é]nervées » qui élèvent leurs enfants seules et finissent par les négliger ou les battre (1988, p. 165), Graham ne peut que condamner la mère de Grégoire :

Qui était-elle pour avoir ainsi rejeté son fils? Que reprochaitelle à son enfant? Graham aurait aimé la rencontrer pour mieux comprendre son jeune ami, mais elle redoutait ses propres réactions. Elle ne pourrait s'empêcher de dire à cette femme qu'elle était une mère indigne. [...] Parfois, elle en venait à souhaiter [que Grégoire] s'installe chez elle pour pouvoir le dorloter, le gâter comme l'enfant qu'il n'avait pas été. (1996, p. 53)

Ici, la mère absente, la mauvaise mère, joue le rôle de repoussoir au profit de l'image maternelle de Graham. Grâce au soutien de celle-ci, au fil de cette série de romans, Grégoire en vient à s'affirmer comme individu, à accepter son homosexualité, à délaisser la prostitution et la cocaïne pour se lancer dans la cuisine et la restauration, à l'instar de son amant, PierreYves, dont on fait la connaissance dans C'est pour mieux t'aimer, mon enfant. 
Comme s'il fallait remplacer un protégé par un autre, dans Soins intensifs, Graham devient la tutrice de l'adolescent Maxime Desrosiers, dont le père, Bruno, est vendeur de drogue et délateur. Lorsque le père est blessé dans un règlement de comptes, on lui amène l'enfant en supposant que « [j]ustement, Graham doit être bonne avec les enfants » (p. 13). Maxime défend son père en soutenant que celui-ci ne le laisse jamais seul plus de deux jours de suite. Sa mère lui manque beaucoup, mais elle est partie vivre avec son nouveau conjoint en Ontario et ne le contacte plus guère. La détective s'indigne d'un tel manque de tendresse maternelle: «Graham éprouvait du ressentiment envers cette inconnue qui abandonnait son fils à un irresponsable. Elle lui rappelait la mère de Grégoire qui avait préféré son amant à son fils. » (p. 44) Dans son rôle de tutrice, Graham remplace la mère indigne en créant «cette étrange famille» (2006, p. 82) constituée de Graham, son amant le médecin légiste Alain Gagnon, Maxime, Grégoire et son chat Léo. Cette famille n'est pas pour autant "nucléaire »; Graham fait appel constamment à son partenaire Rouaix et à sa meilleure amie Léa Boyer pour recevoir des conseils tout en partageant la garde de Maxime avec son père au Saguenay. Maxime passe à travers une adolescence difficile, puisqu'il subit de l'intimidation, une rupture amoureuse et la tentation de la drogue, mais il trouve la sécurité à l'intérieur de cette nouvelle famille, et ce, sans perdre le contact avec son propre père.

Ce que le modèle maternel offert par Maud Graham apporte par rapport à l'ancien modèle nucléaire, c'est la reconnaissance qu'il faut tout un village pour élever un enfant. Sans être mère couveuse, elle offre à Grégoire et à Maxime la sécurité d'une famille recomposée fondée sur l'acceptation de l'individu avec toutes ses faiblesses et ses forces. L'importance 
de cette structure familiale comme élément de rétablissement de l'ordre social est soulignée par la primauté de la réunion familiale en fin de roman où, les malfaiteurs découverts et la justice rendue dans la mesure du possible, Maud Graham se retire avec les membres de sa famille recomposée; c'est le cas du Collectionneur (Maud, Grégoire et Léo), de C'est pour mieux t'aimer, mon enfant (Maud, Alain, Léo et Grégoire), de Silence de mort (Maud, Maxime, Grégoire et Léo), de Sans pardon (Maud, Grégoire et Léo) et de Promesses d'éternité (Maud, Alain, Maxime et Léo). Là où les écrivaines féministes plus radicales choisissent souvent un dénouement ambigu qui subvertit tout retour à un ordre social sécurisant (Desnain, 2009, p. 95), Brouillet rassure les lecteurs en revenant à la cellule familiale, même s'il s'agit d'une famille qui n'est pas comme les autres.

Fidèle à ses principes féministes, Graham fait valoir ses capacités maternelles tout en réclamant le droit des femmes de choisir ou de refuser la maternité :

Léa, sa meilleure amie, affirmait qu'elle était très maternelle, que son attitude envers Maxime en était la preuve parfaite. Qu'il n'était pas nécessaire d'accoucher pour être mère. Et qu'il n'était pas obligatoire d'être mère pour être femme. C'était un choix personnel et Graham avait la chance de vivre dans un pays où les femmes décidaient en toute liberté d'enfanter ou non. (2006, p. 28)

Toujours est-il que Graham se sent coupable de priver son amant Alain, plus jeune qu'elle, des enfants qu'il aurait peutêtre aimé avoir. La pression sociale pour avoir des enfants a diminué au cours des années, mais sans disparaître entièrement. 


\section{"Il faut tout un village »}

Dans cet aperçu du rôle maternel dans les romans de Brouillet, on peut se demander si l'auteure et son personnage ne tombent dans le piège habituel de blâmer la victime, en l'occurrence, la mère. Ceux qui cherchent des solutions plus radicales aux maux sociaux ne les trouveront pas dans cette œuvre, dont les limites sont imposées à la fois par le genre essentiellement conservateur du roman policier et par le féminisme révisionniste. Par contre, bien que Maud Graham ait tendance à blâmer «la mauvaise mère ", elle expose et condamne en même temps le contexte social qui entrave l'agentivité de la mère. La réification $\mathrm{du}$ corps féminin comme réceptacle fertile ou comme assemblage admirable de beaux morceaux de chair peut entraîner la mort spirituelle de la femme et, ainsi, son incompétence maternelle. Dans une structure familiale traditionnelle où le statut social de la mère dépend de celui de son mari ainsi que de la réussite de ses enfants, la mère aura tendance à ne vivre que pour ses enfants, ce qui empêche son épanouissement ainsi que le processus d'individuation de l'enfant. Si la valeur sociale de la femme dépend de sa conformité à l'image de la madone, la mère résistera mal à la tentation d'utiliser son enfant comme faire-valoir. Par contre, selon l'axiologie qui ressort de cette série de romans, la rupture de la famille nucléaire, où les parents divorcés, mais le plus souvent la mère, doivent élever un enfant seul et sans l'appui de la société, ne favorise pas le développement sain de l'enfant. En relevant le défi de materner d'abord Grégoire et ensuite Maxime dans sa vie privée, Maud Graham n'oublie pas l'importance des réformes sociales pour aider les enfants : des centres d'accueil, des programmes pour nourrir les enfants, des 
programmes contre l'intimidation dans les écoles. Ce qu'elle apporte au rôle traditionnel du détective, c'est justement une image de la mère agissante, faisant contraste avec l'archétype de la femme-refuge désincarnée et altruiste, objet d'adoration plutôt que sujet exigeant la transformation du monde. Ce qu'elle apporte à la critique de la société patriarcale, c'est la notion d'une famille où l'institutionnalisation d'une fusion étroite entre l'enfant et la mère cède la place à une vision plutôt communautaire d'une nouvelle famille, basée sur l'appui mutuel libre des préjugés (hétéro)-sexistes de la société bienpensante.

\section{Bibliographie}

Brouillet, Chrystine. (1987), Le Poison dans l'eau, Paris, Denoël/Lacombe.

—. (1988), Préférez-vous les icebergs?, Paris, Denoël/Lacombe.

—. (1995), Le Collectionneur, Montréal, La courte échelle.

—. (1996), C'est pour mieux t'aimer, mon enfant, Montréal, La courte échelle.

—. (1999), Les Fiancées de l'enfer, Montréal, La courte échelle.

—. (2000), Soins intensifs, Montréal, La courte échelle. 
—. (2003), Les Indésirables, Montréal, La courte échelle.

—. (2006), Sans pardon, Montréal, La courte échelle.

—. (2008), Silence de mort, Montréal, La courte échelle.

—. (2009), Promesses d'éternité, Montréal, La courte échelle.

Chodorow, Nancy. (1978), The Reproduction of Mothering: Psychoanalysis and the Sociology of Gender, Berkeley, University of California Press.

DESNAIN, Véronique. (2009), «Gender and Genre: Women in French Crime Writing», dans Claire Gorrara (dir.), French Crime Fiction, Cardiff, University of Wales Press, p. 86-106.

Godin, Colette. (1992-1993), «Vivre de sa plume; l'aventure de Chrystine Brouillet», Femmes d'action, vol. 22, n 2, p. 33-34 et 44.

KLEIN, Kathleen Gregory. (1995 [1988]), The Woman Detective: Gender and Genre, Urbana/Chicago, University of Illinois Press, éd. rev. et corr.

KrISTEVA, Julia. (1980), Pouvoirs de l'horreur, Paris, Seuil.

LACOMBE, Alain. (1975), Le Roman noir américain, Paris, coll. «10$18 »$.

LEMONDE, Anne. (1984), Les Femmes et le roman policier: anatomie d'un paradoxe, Montréal, Québec Amérique.

MunT, Sally. (1994), Murder by the Book? Feminism and the Crime Novel, New York, Routledge. 


\title{
Résumé
}

Kathleen Kellet-Betsos entreprend l'analyse d'une série de roman policiers rattachés peu ou prou au récit de détection classique et qui a connu beaucoup de succès au Québec. Par l'intermédiaire de Maud Graham, détective excentrique, les romans policiers de Chrystine Brouillet contiennent, entre autres, une interrogation féministe des rôles de la mère et de la femme-victime. Kellet-Betsos examine comment les romans de la série déconstruisent les figures traditionnelles de la mère et proposent une nouvelle image du maternalisme.

\begin{abstract}
Kathleen Kellet-Betsos undertakes the analysis of a series of detective novels related to the classic detective genre and which has seen unprecedented success in Québec. Through the intermediary of Maud Graham, an excentric detective, the mystery novels of Chrystine Brouillet offer a feminist interrogation of the roles of mother and woman-victim, among others. Kellet-Betsos examines how the novels in this series deconstruct the traditional figures of the mother and propose a new image of maternalism.
\end{abstract}

